\title{
Review Article \\ Effect of Third-Order Dispersion on the Solitonic Solutions of the Schrödinger Equations with Cubic Nonlinearity
}

\author{
H. Chachou Samet, ${ }^{1}$ M. Benarous, ${ }^{1}$ M. Asad-uz-zaman, ${ }^{2}$ and U. Al Khawaja ${ }^{2}$ \\ ${ }^{1}$ Laboratory for Theoretical Physics and Material Physics, Faculty of Sciences, Hassiba Benbouali University of Chlef, \\ BP 151, 02000 Chlef, Algeria \\ ${ }^{2}$ Physics Department, United Arab Emirates University, P.O. Box 15551, Al-Ain, United Arab Emirates
}

Correspondence should be addressed to H. Chachou Samet; chachou_houria@yahoo.fr

Received 23 March 2014; Revised 25 August 2014; Accepted 28 August 2014; Published 15 September 2014

Academic Editor: Ricardo Weder

\begin{abstract}
Copyright (C) 2014 H. Chachou Samet et al. This is an open access article distributed under the Creative Commons Attribution License, which permits unrestricted use, distribution, and reproduction in any medium, provided the original work is properly cited.
\end{abstract}

\begin{abstract}
We derive the solitonic solution of the nonlinear Schrödinger equation with cubic nonlinearity, complex potentials, and timedependent coefficients using the Darboux transformation. We establish the integrability condition for the most general nonlinear Schrödinger equation with cubic nonlinearity and discuss the effect of the coefficients of the higher-order terms in the solitonic solution. We find that the third-order dispersion term can be used to control the soliton motion without the need for an external potential. We discuss the integrability conditions and find the solitonic solution of some of the well-known nonlinear Schrödinger equations with cubic nonlinearity and time-dependent coefficients. We also investigate the higher-order nonlinear Schrödinger equation with cubic and quintic nonlinearities.
\end{abstract}

\section{Introduction}

The nonlinear Schrödinger equation (NLSE) appears in different branches of physics [1-3]. In optical fiber communication systems, this type of equation describes the dynamics of an optical pulse through the fiber. Due to the advent of high power optical soliton, higher-order terms become very important in describing the soliton propagation accurately through the fiber. The role of the higher-order terms in the stability and dynamics of the solitons thus needs to be understood. To that end, we consider here the most general form of the NLSE with cubic nonlinearity, complex potential, and derivative terms. There are many different approaches to solve the nonlinear partial differential equations, some of which are developed very recently. A few of these approaches are similarity transformation method [4-6], Hirota bilinear method [7], the generalized and improved $\left(G^{\prime} / G\right)$-expansion method $[8,9]$, the mapping method $[10,11]$, and Darboux Transformation (DT) method. We solve this general equation with the DT method. It has the beauty of generating nontrivial solutions from a trivial seed solution of a given integrable NLSE. We discuss the integrability conditions and solve some of the well-known NLSEs with cubic nonlinearity and timedependent coefficients. The effect of the higher-order terms will then be evident. In some cases, we include a linear potential and a term describing the damping or gain in the system.

The key ingredient to solve a NLSE using the DT is a set of matrices, commonly known as the Lax pair. We derive the Lax pair for a given NLSE using a systematic search approach [12]. The aim of this paper is to use the DT to get an exact solitonic solution for the NLSE and study the effect of the higher-order terms in the solution. Specifically, we consider NLSE up to cubic nonlinearity only; some of the well-known NLSEs with cubic nonlinearity are NLSE or Gross-Pitaevskii equation (GPE), Sasa-Satsuma equation (SSE), and Hirota equation (HE). One of the key factors here is that the coefficients are time dependent. The coefficients of different terms in the NLSE can be made time-dependent simply by varying the radius or the nonlinearity along the fiber. Alternatively, the coupling in wave guide arrays may be suitably changed [13]. The time-dependent coefficients introduce a time-dependent amplitude and a position dependent phase, respectively. 
We investigate the integrability conditions of the NLSE with cubic and quintic nonlinearity and find that it is not integrable in the Lax pair sense. Although we manage to find two matrices $\mathbf{U}_{\mathbf{0}}$ and $\mathbf{V}_{\mathbf{0}}$ that generate the given NLSE as their compatibility condition, we were unable to find the full set of matrices for the spectral parameters required to solve the given NLSE using the DT.

The rest of the paper is organized as follows. In Section 2 basic equations for deriving the Lax pair and DT are presented. In Section 3 solutions are found for some well-known NLSEs with cubic nonlinearity. Discussion and conclusions are in Section 4.

\section{Lax Pair and Darboux Transformation}

The Lax pair is the most important ingredient in solving a NLSE using the DT. Here we summarize the basic equations only. In order to derive the Lax pair we start by writing the following two equations:

$$
\begin{gathered}
\Psi_{\mathrm{x}}=\mathrm{U}_{0} \cdot \Psi+\mathrm{U}_{1} \cdot \Psi \cdot \Lambda, \\
\Psi_{\mathrm{t}}=\mathrm{V}_{0} \cdot \Psi+\mathrm{V}_{1} \cdot \Psi \cdot \Lambda+\mathrm{V}_{2} \cdot \Psi \cdot \Lambda^{2}+\mathrm{V}_{3} \cdot \Psi \cdot \Lambda^{3}
\end{gathered}
$$

for an auxiliary field $\Psi$. Here subscripts $\mathbf{t}$ and $\mathbf{x}$ represent the derivative with respect to time and position, respectively. The matrices $\mathrm{U}_{0}, \mathrm{U}_{1}, \mathrm{~V}_{0}, \mathrm{~V}_{1}, \mathrm{~V}_{2}$, and $\mathrm{V}_{3}$ are functions of the solution of the given NLSE and its space derivatives and $\Lambda$ denotes the spectral parameter matrix. The order of the matrices depends on the NLSE to be solved as will be seen next. The compatibility condition $\Psi_{\mathbf{x t}}=\Psi_{\mathbf{t x}}$ leads to the following set of equations:

$$
\begin{gathered}
\mathrm{U}_{0 \mathrm{t}}-\mathrm{V}_{\mathbf{0 x}}+\left[\mathrm{U}_{0}, \mathrm{~V}_{0}\right]=\mathbf{0} \\
\mathrm{U}_{\mathbf{1 t}}-\mathrm{V}_{\mathbf{1 x}}+\left[\mathrm{U}_{0}, \mathrm{~V}_{1}\right]+\left[\mathrm{U}_{1}, \mathrm{~V}_{\mathbf{0}}\right]=\mathbf{0} \\
\mathrm{V}_{2 \mathrm{x}}+\left[\mathrm{V}_{2}, \mathrm{U}_{0}\right]+\left[\mathrm{V}_{1}, \mathrm{U}_{1}\right]=\mathbf{0} \\
\mathrm{V}_{3 \mathrm{x}}+\left[\mathrm{V}_{3}, \mathrm{U}_{0}\right]+\left[\mathrm{V}_{2}, \mathrm{U}_{1}\right]=\mathbf{0} \\
{\left[\mathrm{V}_{3}, \mathrm{U}_{1}\right]=0}
\end{gathered}
$$

where $[\mathbf{X}, \mathbf{Y}]$ denotes the commutator of $\mathbf{X}$ and $\mathbf{Y}$. Equation (2) gives back the original NLSE, which is the condition that the Lax pair must satisfy. The most important and difficult part is to find $\mathbf{U}_{\mathbf{0}}$ and $\mathbf{V}_{\mathbf{0}}$ matrices for a given NLSE. The remaining matrices are determined from $\mathbf{U}_{0}$ and $\mathbf{V}_{\mathbf{0}}$ matrices. We use a systematic search approach to find $\mathbf{U}_{\mathbf{0}}$ and $\mathbf{V}_{\mathbf{0}}$ matrices [12]. It is worth mentioning that for some higherorder NLSE we find $\mathbf{U}_{0}$ and $\mathbf{V}_{\mathbf{0}}$ matrices but do not find the remaining matrices. Therefore, analytical solution of the given higher-order NLSE is not possible using the DT.

After deriving the set of matrices for the given NLSE we use the DT to find the analytic solution. We solve (1) for the auxiliary field $\Psi=\Psi_{\mathbf{0}}$. Using this auxiliary field we find the solution of the original NLSE, which is given by

$$
\mathbf{U}_{0}[\mathbf{1}]=\mathbf{U}_{0}+\left[\mathbf{U}_{1}, \sigma\right]
$$

where $\mathbf{U}_{\mathbf{0}}[\mathbf{1}]$ is the new $\mathbf{U}_{\mathbf{0}}$ matrix and $\sigma=\Psi_{\mathbf{0}} \cdot \boldsymbol{\Lambda} \cdot \boldsymbol{\Psi}_{\mathbf{0}}^{-\mathbf{1}}$. This $\mathrm{U}_{\mathbf{0}}[\mathbf{1}]$ contains the solution of the given NLSE. The beauty of the DT lies in the fact that a trivial seed solution, for example, zero or continuous wave solution of the given NLSE, leads to a nontrivial solution.

\section{NLSE with Cubic Nonlinearity}

In this section we consider the most general NLSE with qubic nonlinearity [1,14-17]. In the presence of damping or gain and external potential, the general NLSE can be written as

$$
\begin{aligned}
i \psi_{t} & +a_{1} \psi_{x x}+a_{2}|\psi|^{2} \psi+i a_{3} \psi_{x x x} \\
& +i a_{4}|\psi|^{2} \psi_{x}+i a_{5} \psi^{2} \psi_{x}^{*}+i \Gamma \psi+V_{\text {ext }} \psi=0
\end{aligned}
$$

In $[18,19]$, the Lax pair of a special case of this equation was found. Here all the coefficients $a_{i} \equiv a_{i}(t)$ are initially assumed to be complex functions of time, but it turns out that integrability restricts them to be real. The functions $V_{\text {ext }} \equiv V_{\text {ext }}(x, t)$ and $\Gamma \equiv \Gamma(t)$ are real and account for the external potential and the damping or gain in the system, respectively. Equation (8) describes the evolution of the electric field envelope of an optical pulse through an optical fiber. The second term denotes the chromatic or group velocity dispersion, the third term denotes the Kerr nonlinearity or self-phase modulation (arises due to intensity dependent refractive index), and the fourth term denotes the third-order dispersion. The fifth and the sixth terms are related to the self-steepening and self-frequency shift due to the Raman scattering.

Although we have found two $2 \times 2$ matrices for $\mathbf{U}_{0}$ and $\mathbf{V}_{0}$, other integrability conditions do come in while deriving the remaining matrices; in addition to that we need to use $3 \times 3$ matrices. Employing the systematic search approach we find the complete set of matrices for (8), which is rendered to the Appendix for convenience.

When we use $\mathbf{U}_{\mathbf{0}}$ and $\mathbf{V}_{\mathbf{0}}$ in (2) we get back (8) with

$$
\begin{gathered}
\Gamma=\frac{\dot{a}_{2}}{2 a_{2}}-\frac{\dot{a}_{1}}{2 a_{1}}, \\
V_{\text {ext }}=\frac{a_{3} \dot{a}_{1}-a_{1} \dot{a}_{3}}{3 a_{3}^{2}} x+\frac{2 a_{1}^{3}}{27 a_{3}^{2}} .
\end{gathered}
$$

The remaining two integrability conditions are

$$
a_{4}=\frac{9 a_{2} a_{3}}{2 a_{1}}, \quad a_{5}=\frac{3 a_{2} a_{3}}{2 a_{1}} .
$$

Therefore (8) is integrable only with three independent parameters $a_{1}, a_{2}$, and $a_{3}$. One point worth mentioning here is that the damping or gain, $\Gamma$, and the linear part of the potential, $V_{\text {ext }}$, arise due to the time variation of the coefficients $a_{1}, a_{2}$, and $a_{3}$.

Applying the DT on the Lax pair of (8) the following solitonic solution can be derived:

$$
\psi(x, t)=\frac{A}{\cosh (2 x-b)} e^{i\left(a_{1} / 3 a_{3}\right)(x+\pi / 2)},
$$

where $A=2 \sqrt{a_{1} / a_{2}}$ and

$$
b=\int\left(\frac{2 a_{1}^{2}}{3 a_{3}}+8 a_{3}\right) d t-\frac{1}{2} \log 2 .
$$




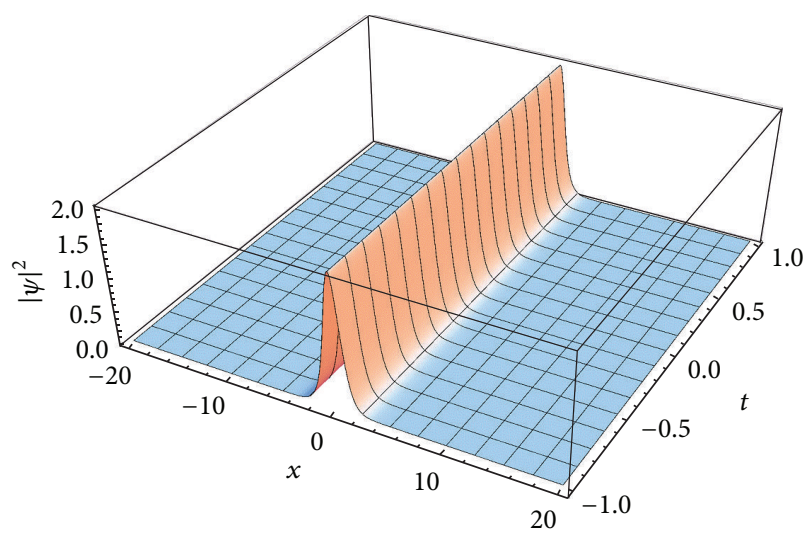

(a)

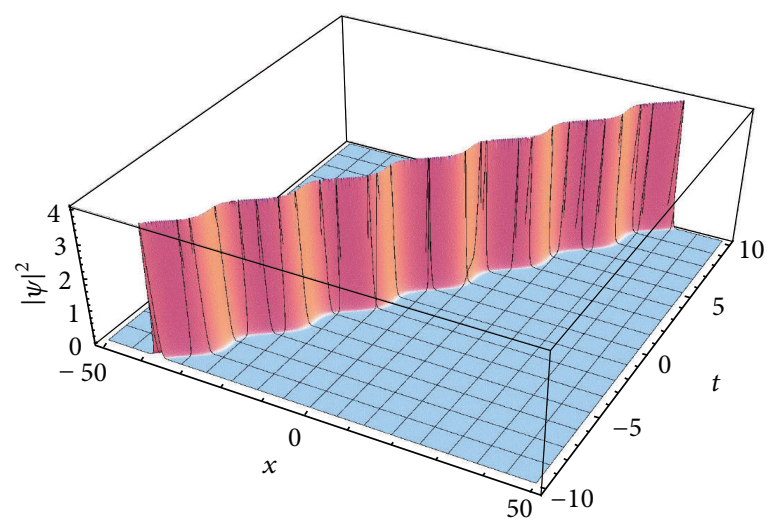

(c)

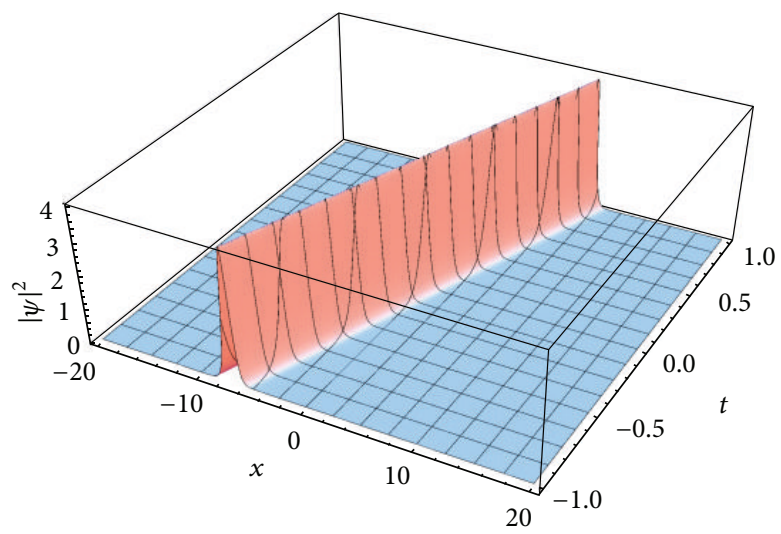

(b)

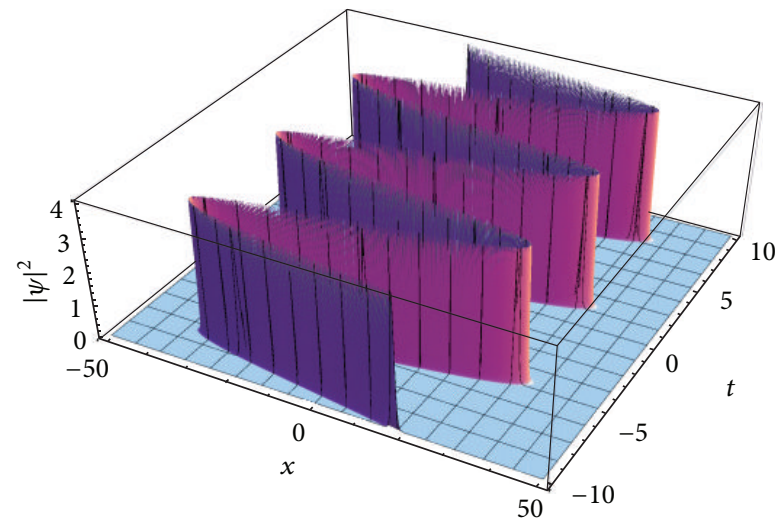

(d)

FIGURE 1: (a) Solitonic solution of NSLE with constant coefficients, $a_{1}=1$ and $a_{2}=1$ (color online). (b) The solution of (8) with constant coefficients, namely, $a_{1}=1, a_{2}=1$, and $a_{3}=0.05$. (c) Trigonometric time dependence for $a_{1}=\cos (t), a_{2}=\cos (t)$, and constant third-order dispersion $a_{3}=0.05$. (d) All the three coefficients are trigonometrically time dependent $a_{1}=\cos (t), a_{2}=\cos (t)$, and $a_{3}=0.05 \cos (t)$.

The amplitude $A$ depends on the ratio of the coefficients of the chromatic dispersion and the nonlinearity. The phase is position dependent and depends on the ratio of the coefficients of the chromatic and third-order dispersions. For repulsive interaction, that is, $a_{2}<0$, the equation supports diverging solutions, one of which is given by

$$
\psi(x, t)=\frac{A}{\sinh (2 x-b)} e^{i\left(a_{1} / 3 a_{3}\right)(x+\pi / 2)},
$$

where $A=2 \sqrt{-a_{1} / a_{2}}$ and $b$ is given by (12).

In both the attractive and repulsive cases the third-order dispersion coefficient controls the phase of the envelope. One important factor to note that the self-steepening and the Raman scattering terms arise due to the third-order dispersion through the relations given by (10). The group velocity of the envelope depends on the coefficients $a_{1}$ and $a_{3}$. Therefore, we can control the group velocity, that is, the center of mass motion of the soliton, by properly choosing the chromatic dispersion and the third-order dispersion. This interesting fact can be used to manipulate soliton motion without the need of an external potential.

Figure 1 shows the solitonic solutions given by (11). For comparison, Figure 1(a) shows the solitonic solution of NLSE with constant coefficients, that is, without any damping and external potential. In Figure 1(b), we choose all the three coefficients $a_{1}-a_{3}$ to be time independent and take $a_{3}$ to be very small compared to $a_{1}$ and $a_{2}$. In this particular case $\Gamma=0$ and $V_{\text {ext }}=2 a_{1}^{2} / 27 a_{3}^{2}$ and the center of mass velocity of the soliton is a constant. We assume trigonometric time dependence for $a_{1}$ and $a_{2}$ [20] and constant $a_{3}$ in Figure 1(c). The soliton moves with a constant velocity with oscillation around it. In Figure 1(d), we assume all the coefficients be trigonometrically time dependent and the center of mass velocity oscillates. From Figures 1(b)-1(c), it is obvious that the center of mass velocity can be controlled by appropriate choice of the third-order dispersion coefficient $a_{3}$.

\subsection{Special Cases}

3.1.1. Nonlinear Schrödinger Equation, NLSE. If we set $a_{3}=$ $a_{4}=a_{5}=0$ in (8) we get the standard NLSE or GPE with damping or gaining and potential, which can be written as

$$
i \psi_{t}+a_{1} \psi_{x x}+a_{2}|\psi|^{2} \psi+i \Gamma \psi+V_{\text {ext }} \psi=0
$$

This is one of the most well-known NLSEs in the optical [1], Bose-Einstein [2], and ocean wave communities [3]. 
The Lax pair for (14) with time-independent coefficient and without damping and potential was first found by Zakharov and Shabat [21] and was then widely used in the Darboux transformation to find exact solutions. The damping is given by $\Gamma=\dot{a}_{2} /\left(2 a_{2}\right)-\dot{a}_{1} /\left(2 a_{1}\right)$ and the potential is given by $V_{\text {ext }}=c_{1}^{2} a_{1}-\dot{c}_{1} x$. Equation (14) supports solitonic solutions and one such solution is given by

$$
\psi(x, t)=\frac{A}{\cosh \left[c_{3} x+b\right]} e^{i\left[c_{3}^{2} \int a_{1} d t-c_{1} x\right]},
$$

where $A=-4 c_{3} \sqrt{2 a_{1} / a_{2}}$ and $b=\sqrt{2} c_{3} \int c_{1} a_{1} d t$. Similar results are also found by Kumar et al. [22]. Al Khawaja found solution of (14) with quadratic potential, periodic potential, and different types of solution with linear potential.

It is well-known that the NLSE or GPE with timedependent coefficients is integrable with potential up to quadratic power in $x[23,24]$. Here, we find the Lax pair for NLSE with linear potential $V_{\text {ext }}=c_{1}^{2} a_{1}-\dot{c}_{1} x$; this is due to the fact that we derive the Lax pair for the NLSE from that of the higher-order NLSE with cubic-quintic nonlinearity by taking appropriate limit of $a_{3}=a_{4}=a_{5}=a_{6}=0$ simultaneously [see (21)]. The higher-order terms in higher-order NLSE restrict integrability to linear potentials. One of the original motivations of our work is to solve the most general NLSE with cubic-quintic nonlinearity and find the solutions for other well-known NLSEs as special cases. However, we find that general NLSE with cubic-quintic nonlinearity is not solvable in the Lax pair sense.

3.1.2. Hirota Equation, $H E$. If we set $a_{5}=0$ in (8) we get the HE [25]; namely,

$$
\begin{aligned}
i \psi_{t} & +a_{1} \psi_{x x}+a_{2}|\psi|^{2} \psi+i a_{3} \psi_{x x x} \\
& +i a_{4}|\psi|^{2} \psi_{x}+i \Gamma \psi+V_{\text {ext }} \psi=0
\end{aligned}
$$

In this case we find that the equation is integrable only if $a_{4}=$ $3 a_{2} a_{3} / a_{1}, \Gamma=\dot{a}_{4} /\left(2 a_{4}\right)-\dot{a}_{3} /\left(2 a_{3}\right)$, and $V_{\text {ext }}=0$. The onesoliton solution of the HE is given by

$$
\psi(x, t)=\frac{A}{\cosh [\sqrt{6} x-b]} e^{6 i \int a_{1} d t},
$$

where $A=-12 \sqrt{a_{3} / a_{4}}$ and $b=6 \sqrt{6} \int a_{3} d t$. Similar result was also found by Dai and Zhang [26].

3.1.3. Sasa-Satsuma Equation, SSE. If we set $a_{1}=a_{2}=0$ in (8) we get the SSE [27]; namely,

$$
\begin{aligned}
i \psi_{t} & +i a_{3} \psi_{x x x}+i a_{4}|\psi|^{2} \psi_{x} \\
& +i a_{5} \psi^{2} \psi_{x}^{*}+i \Gamma \psi+V_{\text {ext }} \psi=0 .
\end{aligned}
$$

We find that the SSE is integrable for arbitrary values of $a_{3}$, $a_{4}$, and $a_{5}$ with $a_{4} \neq 2 a_{5}$ and $\Gamma=V_{\text {ext }}=0$. The one-soliton solution of the SSE is given by

$$
\psi(x, t)=\frac{i A}{\cosh \left(2 c_{2} \lambda x-b\right)},
$$

where $A=2 c_{1} c_{2} \lambda_{3} / c_{3}$ and $b=8 c_{2}^{3}(t-1) \lambda^{3} a_{3}$. Similar result was found by Gilson et al. [28].

We further investigate the integrability of the general higher-order NLSE equation with cubic and quintic nonlinearities [29-31]. In nonlinear optics, the nonlinear Schrödinger equation with quintic nonlinearity becomes important as the intensity and the inverse width of the optical pulse increase. In the presence of damping or gaining and an external potential, in nonlinear optics the general NLSE with cubic and quintic nonlinearities can be written as

$$
\begin{aligned}
i \psi_{t} & +a_{1} \psi_{x x}+a_{2}|\psi|^{2} \psi+i a_{3} \psi_{x x x} \\
& +i a_{4}|\psi|^{2} \psi_{x}+i a_{5} \psi^{2} \psi_{x}^{*} \\
& +a_{6}|\psi|^{4} \psi+i a_{7}|\psi|^{4} \psi_{x}+i a_{8}|\psi|^{2} \psi^{2} \psi_{x}^{*} \\
& +i \Gamma \psi+V_{\text {ext }} \psi=0 .
\end{aligned}
$$

Here, as for (8), all the coefficients $a_{i}(t)$ turn out to be real. The terms with coefficients $a_{6}, a_{7}$, and $a_{8}$ account for the quintic nonlinearity and are generally known as non-Kerr nonlinearity. All the other terms are similar to those for (8). Using the systematic search approach we find the Lax pair for (20), which is given by

$$
\begin{gathered}
\mathbf{U}_{\mathbf{0}}=\left(\begin{array}{cc}
c_{1} & i c_{2} e^{G} \psi \\
-i c_{2} e^{-G} \psi^{*} & c_{1}
\end{array}\right), \\
\mathbf{V}_{\mathbf{0}}=\left(\begin{array}{ll}
v_{11} & v_{12} \\
v_{21} & v_{22}
\end{array}\right),
\end{gathered}
$$

where the matrix elements $v_{i j}$ are given by

$$
\begin{gathered}
v_{11}=-i c_{5}|\psi|^{2}+c_{2}^{2} a_{3}\left(\psi^{*} \psi_{x}-\psi \psi_{x}^{*}\right), \\
v_{12}=e^{G}\left[i \frac{c_{4} c_{5}}{c_{2}} \psi-\frac{c_{5}}{c_{2}} \psi_{x}-i c_{2} a_{3} \psi_{x x}\right. \\
\left.-\frac{i}{3} c_{2}\left(a_{4}+a_{5}\right)|\psi|^{2} \psi-\frac{i}{3} c_{2} a_{7}|\psi|^{4} \psi\right], \\
v_{21}=e^{-G}\left[-i \frac{c_{4} c_{5}}{c_{2}} \psi^{*}-\frac{c_{5}}{c_{2}} \psi_{x}^{*}+i c_{2} a_{3} \psi_{x x}^{*}\right. \\
\left.+\frac{i}{3} c_{2}\left(a_{4}+a_{5}\right)|\psi|^{2} \psi^{*}+\frac{i}{3} c_{2} a_{7}|\psi|^{4} \psi^{*}\right], \\
v_{22}= \\
i c_{5}|\psi|^{2}-c_{2}^{2} a_{3}\left(\psi^{*} \psi_{x}-\psi \psi_{x}^{*}\right) .
\end{gathered}
$$

Here $c_{2}=\sqrt{\left(2 a_{5}-a_{4}\right) / 6 a_{3}}, c_{4}=-3 a_{6} / a_{7}, c_{5}=c_{2}^{2}\left[a_{1}\left(a_{4}+\right.\right.$ $\left.\left.a_{5}\right)-3 a_{2} a_{3}\right] /\left(3 a_{5}\right)$, and $G=c_{3}+i c_{4} x$, and $c_{1}$ and $c_{3}$ are two arbitrary time-independent constants. Using (21) in (2) we get (20) with the following integrability conditions: $a_{7}=$ $9 a_{3} a_{5} a_{6} /\left(3 a_{2} a_{3}-a_{1} a_{4}+2 a_{1} a_{5}\right), a_{8}=2 a_{7} / 3, \Gamma=\dot{c}_{2} / c_{2}$, and $V_{\text {ext }}=c_{4}^{2} c_{5} / c_{2}^{2}-\dot{c}_{4} x$. This Lax pair is a fake one as we could not find the other matrices to solve the spectral problem given by (20). Therefore (20) is not integrable in the Lax pair sense. We also tried $3 \times 3$ matrices for $\mathbf{U}_{0}$ and $\mathbf{V}_{0}$, as we did for the NLSE with cubic nonlinearity, but we were unable to find the other matrices necessary to solve the spectral problem. 


\section{Conclusion}

We employ the Darboux transformation to solve some of the NLSEs with cubic nonlinearity and time-dependent coefficients. We investigate the integrability of NLSE with damping and linear potential and find that it is integrable only with three independent coefficients, namely, dispersion, Kerr nonlinearity, and third-order dispersion coefficients. The coefficients for the Raman and self-steepening terms depend on the other three coefficients. The damping and linear part of the potential arise due to the time variation of the coefficients. This equation supports solitonic solution for focusing nonlinearity. For defocusing nonlinearity diverging nonsolitonic solution exists. In both the cases the coefficient for the third-order dispersion can control the center of mass motion of the solutions. This finding can be used to control the motion of the soliton in the optical fiber without an external potential.

Using the same technique we are able to find the solitonic solution for some of the well-known NLSEs, namely, Hirota, Sasa-Satsuma, and Nonlinear Schrödinger equations. The Hirota and Sasa-Satsuma equations are not integrable with a linear potential, though the former is integrable with timedependent coefficients. We check that the solutions of these equations are similar to those already found in the literature.

We also investigate the integrability conditions for the NLSE with cubic and quintic nonlinearities, derivative terms, and complex potential. In this case, only fake Lax pairs were found indicating the nonintegrability of the equation in the Lax pair sense.

\section{Appendix}

The Lax Pair of (8). Lising the lax-pair-search method described in Section 2, we find sets of all matrices defining the Lax pair of (8):

$$
\begin{gathered}
\mathbf{U}_{\mathbf{0}}=\left(\begin{array}{ccc}
0 & -c_{3} e^{-G} \psi & c_{3} e^{G} \psi^{*} \\
c_{3} e^{G} \psi^{*} & 0 & 0 \\
-c_{3} e^{-G} \psi & 0 & 0
\end{array}\right), \\
\mathbf{U}_{\mathbf{1}}=\left(\begin{array}{ccc}
c_{1} & 0 & 0 \\
0 & c_{1}+c_{2} & 0 \\
0 & 0 & c_{1}+c_{2}
\end{array}\right), \\
\mathbf{V}_{\mathbf{0}}=\left(\begin{array}{lll}
v_{011} & v_{012} & v_{013} \\
v_{021} & v_{022} & v_{023} \\
v_{031} & v_{032} & v_{033}
\end{array}\right),
\end{gathered}
$$

where

$$
\begin{gathered}
v_{011}=v_{023}=v_{032}=0, \\
v_{012}=v_{031}=\frac{i c_{3}^{3} e^{-G}\left(Q_{1}+Q_{2}\right)}{9 \sqrt{2} a_{2} a_{3}}, \\
v_{013}=v_{021}=\frac{i c_{3}^{3} e^{-G}\left(Q_{1}^{*}+Q_{2}^{*}\right)}{9 \sqrt{2} a_{2} a_{3}},
\end{gathered}
$$

$$
\begin{aligned}
& v_{022}=\frac{a_{2}}{6 a_{1}}\left(3 a_{3}\left[\psi^{*} \psi_{x}-\psi \psi_{x}^{*}\right]-2 i a_{1}|\psi|^{2}\right), \\
& v_{033}=\frac{a_{2}}{6 a_{1}}\left(3 a_{3}\left[\psi \psi_{x}^{*}-\psi_{x} \psi^{*}\right]+2 i a_{1}|\psi|^{2}\right) .
\end{aligned}
$$

Here

$$
\begin{gathered}
Q_{1}=18 a_{2} a_{3}^{2}|\psi|^{2} \psi+2 a_{1}^{3} \psi, \\
Q_{2}=9 a_{1} a_{3}^{2} \psi_{x x}-6 i a_{1}^{2} a_{3} \psi_{x}, \\
\mathbf{V}_{1}=\left(\begin{array}{lll}
v_{111} & v_{112} & v_{113} \\
v_{121} & v_{122} & v_{123} \\
v_{131} & v_{132} & v_{133}
\end{array}\right), \\
v_{111}=\frac{c_{2} a_{2} a_{3}|\psi|^{2}}{a_{1}}+\frac{c_{2} a_{1}^{2}}{6 a_{3}}, \\
v_{112}=\frac{1}{3 \sqrt{2}} c_{2} c_{3} e^{-G}\left(a_{1} \psi+3 i a_{3} \psi_{x}\right) \\
v_{113}=\frac{1}{3 \sqrt{2}} c_{2} c_{3} e^{G}\left(a_{1} \psi^{*}-3 i a_{3} \psi_{x}^{*}\right) \\
v_{121}=-\frac{1}{3 \sqrt{2}} c_{2} c_{3} e^{G}\left(a_{1} \psi^{*}-3 i a_{3} \psi_{x}^{*}\right) \\
v_{122}=-\frac{c_{2}}{6 a_{1} a_{3}}\left(a_{1}^{3}+3 a_{2} a_{3}^{2}|\psi|^{2}\right) \\
v_{123}=\frac{c_{2}}{2 a_{1}} e^{2 G} a_{2} a_{3}\left(\psi^{*}\right)^{2} \\
v_{133}=-\frac{1}{6 a_{1} a_{3}}\left(a_{1}^{3}+3 a_{2} a_{3}^{2}|\psi|^{2}\right) \\
v_{132}=\frac{c_{2}}{2 a_{1}} e^{--G} e^{-G}\left(a_{1} \psi+3 i a_{3} \psi_{x}\right) \\
v_{3} \psi^{2}
\end{gathered}
$$

$\mathrm{V}_{2}$

$$
\begin{gathered}
=\left(\begin{array}{ccc}
c_{4} & -i c_{2}^{2} c_{3} a_{3} e^{-G} \psi & i c_{2}^{2} c_{3} a_{3} e^{G} \psi^{*} \\
i c_{2}^{2} c_{3} a_{3} e^{G} \psi^{*} & c_{4} & 0 \\
-i c_{2}^{2} c_{3} a_{3} e^{-G} \psi & 0 & c_{4}
\end{array}\right), \\
\mathbf{V}_{3}=\left(\begin{array}{cccc}
c_{2}^{3} a_{3}+c_{5} & 0 & \\
0 & c_{5} & 0 \\
0 & 0 & c_{5}
\end{array}\right) .
\end{gathered}
$$

Here, $c_{2}=\sqrt{2 a_{5}-a_{4} /\left(6 a_{3}\right)}, c_{3}=\sqrt{a_{5} /\left(2 a_{3}\right)}, c_{5}=-\left(2 c_{2}^{2} a_{1}+\right.$ $\left.a_{2}\right) / a_{5}$, and $G=i a_{1} x /\left(3 a_{3}\right)$, and $c_{1}$ is an arbitrary timeindependent constant.

\section{Conflict of Interests}

We certify that there is no conflict of interests with any financial organization regarding the material discussed in the paper. 


\section{Acknowledgments}

The authors H. Chachou Samet and M. Benarous acknowledge the support provided by Hassiba Benbouali University of Chlef, Algeria. The authors M. Asad-uz-zaman and U. Al Khawaja acknowledge the support provided by the United Arab Emirates University under the Grant UAEUNRF 2011 and the support provided by King Fahd University of Petroleum and Minerals under Group Project nos. RG11071, RG1107-2, RG1214-1, and RG1214-2.

\section{References}

[1] A. Hasegawa and Y. Kodama, Solitons in Optical Communincations, Oxford University Press, New York, NY, USA, 1995.

[2] C. J. Pethick and H. Smith, Bose-Einstein Condensation in Dilute Gases, Cambridge University Press, 2002.

[3] C. Kharif, E. Pelinovsky, and A. Slunyaev, Rogue Waves in the Ocean, Springer, 2010.

[4] C. Q. Dai, X. G. Wang, and G. Q. Zhou, "Stable light-bullet solutions in the harmonic and parity-time-symmetric potentials," Physical Review A, vol. 89, Article ID 013834, 2014.

[5] C. Q. Dai and H. P. Zhu, "Superposed Kuznetsov-Ma solitons in a two-dimensional graded-index grating waveguide," Journal of the Optical Society of America B, vol. 30, pp. 3291-3297, 2013.

[6] C.-Q. Dai and H.-P. Zhu, "Superposed Akhmediev breather of the $(3+1)$-dimensional generalized nonlinear Schrödinger equation with external potentials," Annals of Physics, vol. 341, pp. 142-152, 2014.

[7] W. J. Liu, B. Tian, and M. Lei, "Elastic and inelastic interactions between optical spatial solitons in nonlinear optics," Laser Physics, vol. 23, no. 9, Article ID 095401, 2013.

[8] H. Naher, F. A. Abdullah, and M. A. Akbar, "Generalized and Improved $\left(G^{\prime} / G\right)$-Expansion Method for (3+1)-Dimensional Modified KdV-Zakharov-Kuznetsev Equation," PLoS ONE, vol. 8, no. 5, Article ID e64618, 2013.

[9] H. Naher and F. A. Abdullah, "Further extension of the generalized and improved $\left(G^{\prime} / G\right)$-expansion method for nonlinear evolution equation," Journal of the Association of Arab Universities for Basic and Applied Sciences. In press.

[10] Y. Wang and C. Dai, "Elastic interactions between multi-valued foldons and anti-foldons for the $(2+1)$-dimensional variable coefficient Broer-Kaup system in water waves," Nonlinear Dynamics, vol. 74, no. 1-2, pp. 429-438, 2013.

[11] Transformations and Solitons, Springer Series in Nonlinear Dynamics, Springer, Berlin, Germany, 1991.

[12] U. Al Khawaja, "Lax pairs of time-dependent Gross-Pitaevskii equation," Journal of Physics A, vol. 39, no. 31, pp. 9679-9691, 2006.

[13] A. Szameit and S. Nolte, "Discrete optics in femtosecond-laserwritten photonic structures," Journal of Physics B, vol. 43, Article ID 163001, 2010.

[14] Y. Kodama, "Optical solitons in a monomode fiber," Journal of Statistical Physics, vol. 39, no. 5-6, pp. 597-614, 1985.

[15] Y. Kodama and A. Hasegawa, "Nonlinear pulse propagation in a monomode dielectric guide," IEEE Journal of Quantum Electronics, vol. 23, no. 5, pp. 510-524, 1987.

[16] M. J. Potasek, "Novel femtosecond solitons in optical fibers, photonic switching, and computing," Journal of Applied Physics, vol. 65 , no. 3, pp. 941-953, 1989.
[17] B. Tian, Y.-T. Gao, and H.-W. Zhu, "Variable-coefficient higherorder nonlinear Schrödinger model in optical fibers: Variablecoefficient bilinear form, Bäcklund transformation, brightons and symbolic computation," Physics Letters A, vol. 366, no. 3, pp. 223-229, 2007.

[18] K. Porsezian and K. Nakkeeran, "Optical solitons in presence of Kerr dispersion and self-frequency shift," Physical Review Letters, vol. 76, no. 21, pp. 3955-3958, 1996.

[19] A. Mahalingam and K. Porsezian, "Propagation of dark solitons with higher-order effects in optical fibers," Physical Review E, vol. 64, Article ID 046608, 2001.

[20] W.-P. Zhong, M. R. Belic, Y. Lu, and T. Huang, "Traveling and solitary wave solutions to the one-dimensional Gross-Pitaevskii equation," Physical Review E, vol. 81, Article ID 016605, 2010.

[21] V. E. Zakharov and A. B. Shabat, "Tonkaya teoriya dvumernoi samofokusirovki i odnomernoi avtomodulyatsii voln $\mathrm{v}$ nelineinykh sredakh," Zhurnal Eksperimental'noi $i$ Teoreticheskoi Fiziki, vol. 61, pp. 118-134, 1971.

[22] H. Kumar, A. Malik, and F. Chand, "Soliton solutions of some nonlinear evolution equations with time-dependent coefficients," Pramana-Journal of Physics, vol. 80, no. 2, pp. 361367, 2013.

[23] V. N. Serkin, A. Hasegawa, and T. L. Belyaeva, "Nonautonomous solitons in external potentials," Physical Review Letters, vol. 98, Article ID 074102, 2007.

[24] U. Al Khawaja, "A comparative analysis of Painlevé, Lax pair, and similarity transformation methods in obtaining the integrability conditions of nonlinear Schrödinger equations," Journal of Mathematical Physics, vol. 51, no. 5, Article ID 053506, 11 pages, 2010.

[25] R. Hirota, "Exact envelope-soliton solutions of a nonlinear wave equation," Journal of Mathematical Physics, vol. 14, pp. 805-809, 1973.

[26] C. Dai and J. Zhang, "New solitons for the Hirota equation and generalized higher-order nonlinear Schrodinger equation with variable coefficients," Journal of Physics A Mathematical and General, vol. 39, no. 4, pp. 723-737, 2006.

[27] N. Sasa and J. Satsuma, "New-type of soliton solutions for a higher-order nonlinear Schrödinger equation," Journal of the Physical Society of Japan, vol. 60, no. 2, pp. 409-417, 1991.

[28] C. Gilson, J. Hietarinta, J. Nimmo, and Y. Ohta, "Sasa-Satsuma higher-order nonlinear Schrödinger equation and its bilinearization and multisoliton solutions," Physical Review E, vol. 68, Article ID 016614, 2003.

[29] H. Triki and A. Biswas, "Soliton solutions for a generalized fifthorder KdV equation with $t$-dependent coefficients," Waves in Random and Complex Media, vol. 21, no. 1, pp. 151-160, 2011.

[30] A. Choudhuri and K. Porsezian, "Dark-in-the-Bright solitary wave solution of higher-order nonlinear Schrödinger equation with non-Kerr terms," Optics Communications, vol. 285, no. 3, pp. 364-367, 2012.

[31] A. Choudhuri and K. Porsezian, "Higher-order nonlinear Schrödinger equation with derivative non-Kerr nonlinear terms: a model for sub-10-fs-pulse propagation," Physical Review A, vol. 88, Article ID 033808, 2013. 


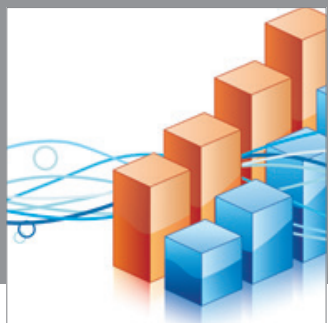

Advances in

Operations Research

mansans

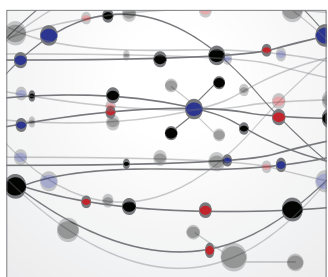

The Scientific World Journal
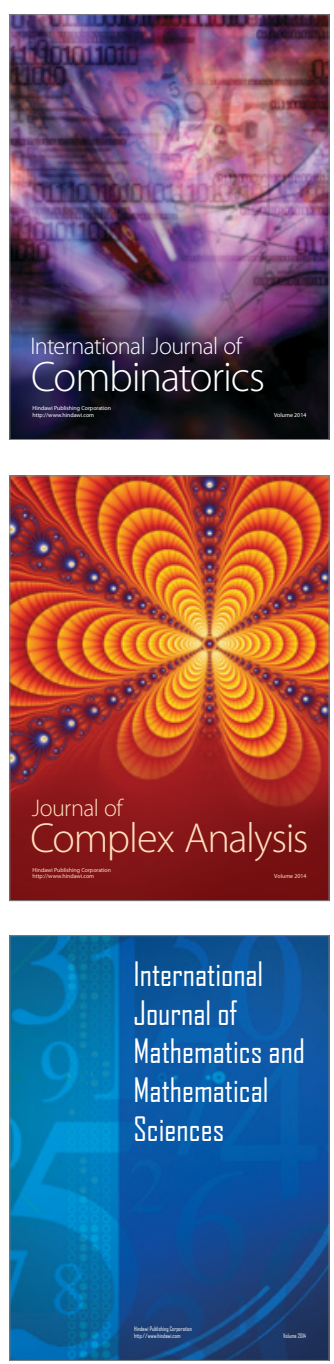
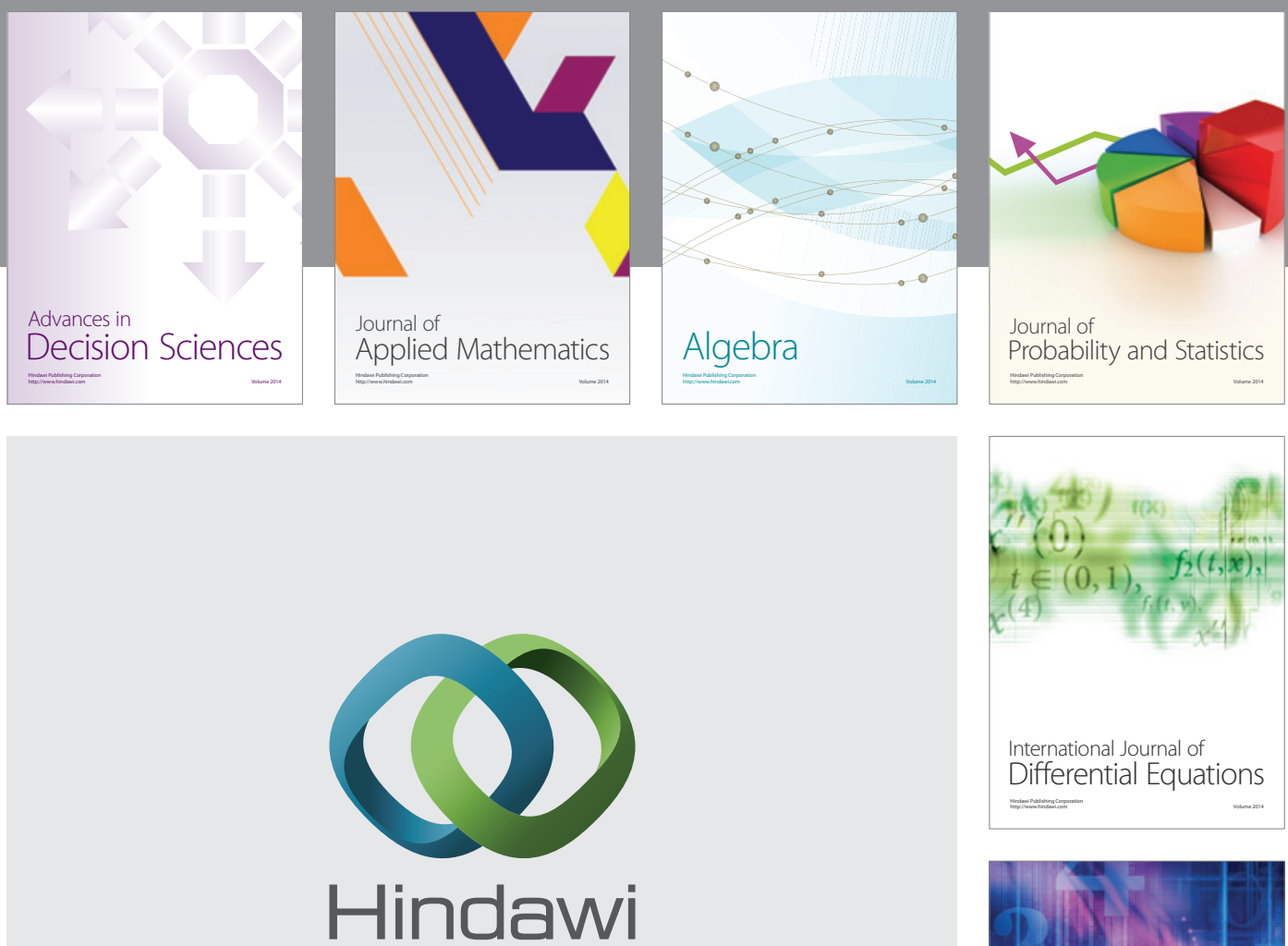

Submit your manuscripts at http://www.hindawi.com
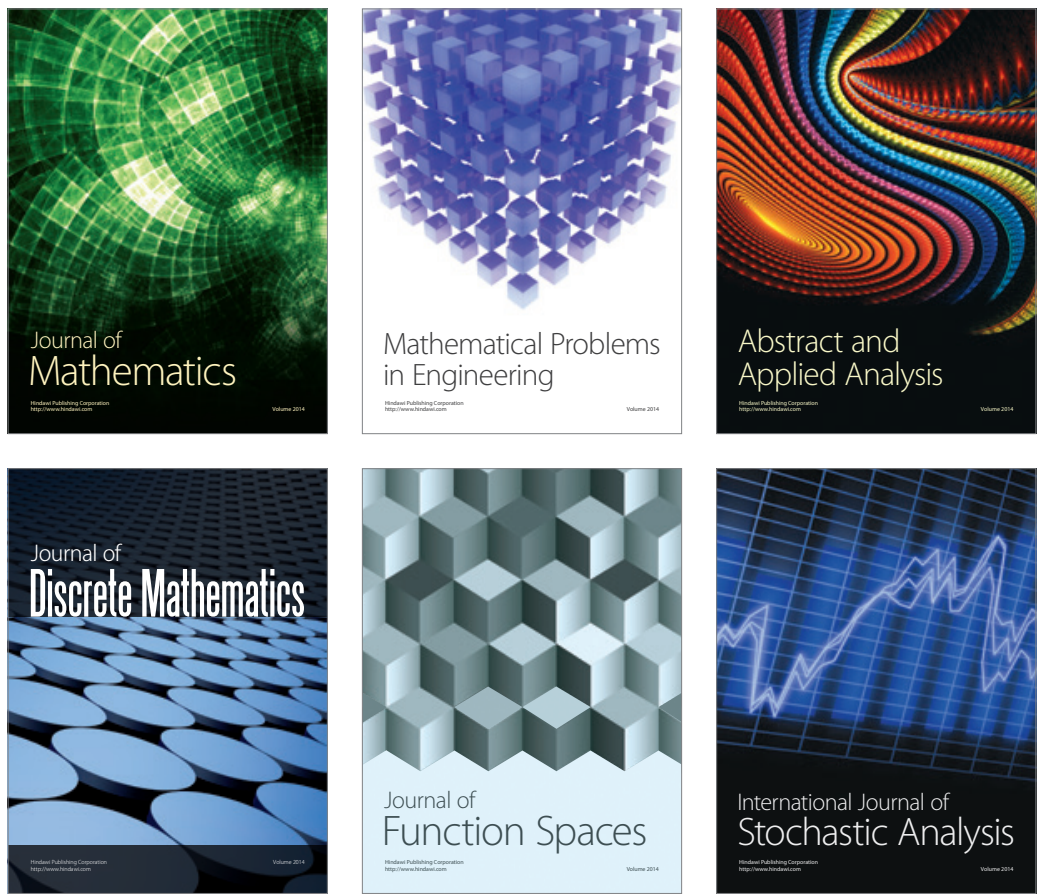

Journal of

Function Spaces

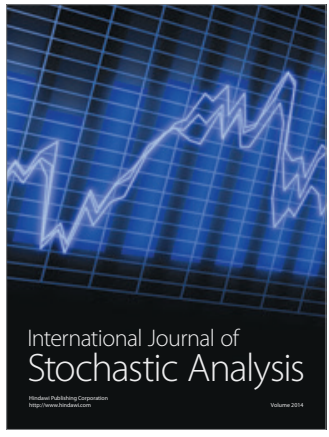

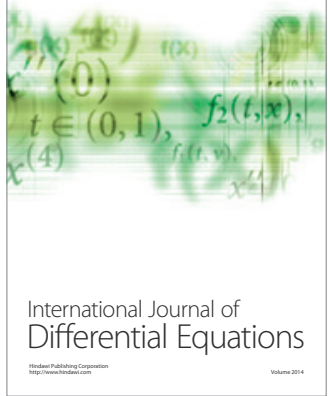
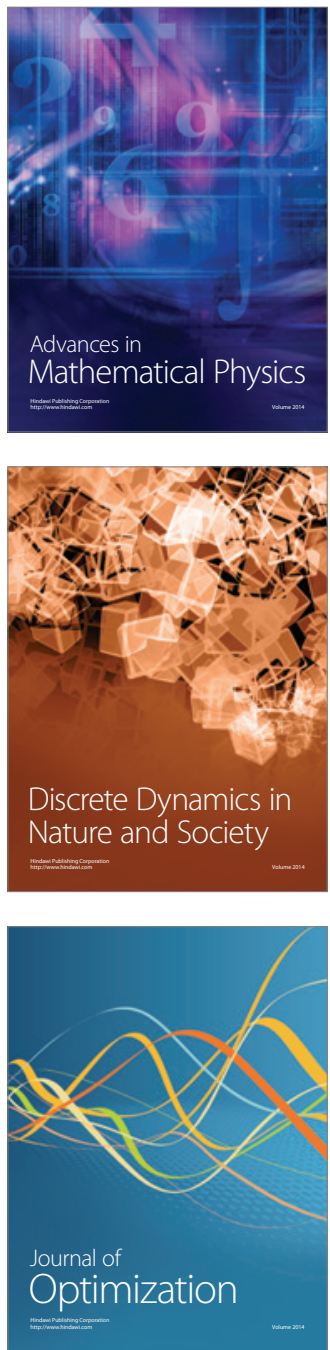